\title{
Small Occupancy Robotic Mechanisms for Endoscopic Surgery
}

\author{
Yuki Kobayashi, Shingo Chiyoda, Kouichi Watabe, \\ Masafumi Okada, and Yoshihiko Nakamura \\ Department of Mechano-Informatics, \\ The University of Tokyo, \\ 7-3-1, Hongo, Bunkyoku, Tokyo 113-8656 Japan, \\ nakamura@ynl.t.u-tokyo.ac.jp \\ http://www.ynl.t.u-tokyo.ac.jp/index.html
}

\begin{abstract}
To make the endoscopic surgery more precise and more accessible, computer-enhanced surgical robot systems are introduced and accepted in the surgical community. Present surgical robot systems unfortunately occupy a significant amount of space in operating rooms. It sometimes prohibits surgeons from emergency access to the patient. In this paper, we propose a design of small occupancy robots for endoscopic surgery. The design concept consists of three components. Namely, the Active Forceps, the Active Trocar, and the passive positioner. The detailed design of the Active Forceps and the Active Trocar presented, and the performance of prototypes are also reported.
\end{abstract}

\section{Introduction}

With the developments of robot assisted surgery system, various new procedures of minimally invasive surgery become possible [1] [2] [3] [4. It enables surgeons to perform surgery with high precision and smaller incisions so that the patient's trauma is reduced, scarring is minimized, and recovery is accelerated. Although several surgical robots are currently in use, they unfortunately occupy a significant amount of space in operating rooms. The manipulators stretched over patients sometimes prohibit surgeons from accessing patients in an emergency. This is due to the fact that the efforts of miniaturization are focused only on the forceps and hardly made for robotic mechanisms so far. The long arm needs high stiffness to move precisely, and the high stiffness makes the mechanism large and heavy. Therefore the robotic system for endoscopic surgery occupy a large space in operating rooms. In this paper, we propose design concepts of small occupancy robots for endoscopic surgery which has six degree-of-freedom. The efforts to make the surgical robot system small also realize mechanical stiffness and response.

\section{Small Occupancy Robotic System}

\subsection{Design Specification}

During endoscopic surgery, surgical instruments move focusing on the trocar port. As a way surgeon holds a surgical instrument in the endoscopic surgery,

T. Dohi and R. Kikinis (Eds.): MICCAI 2002, LNCS 2488, pp. 75-82 2002.

(C) Springer-Verlag Berlin Heidelberg 2002 


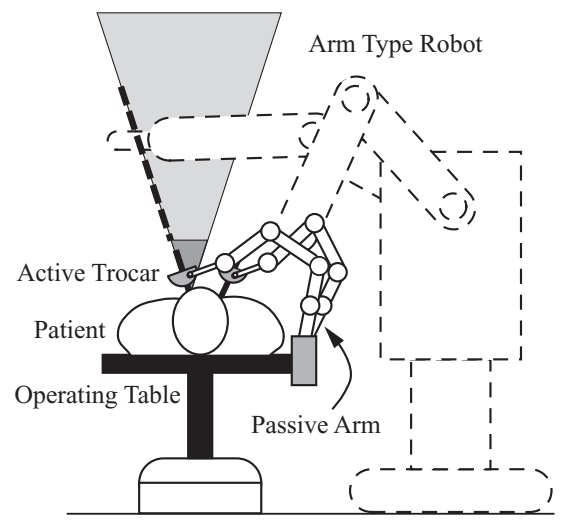

Fig. 1. Area Occupancy of the Surgical Robots

the surgical robot with arm shown on the right of Figure 1 with the dashed lines holds the back end of an surgical instrument. With this mechanism, a surgical robot moves, occupying large amount of space above of the patient. To miniaturize the area occupancy of the surgical robot, we propose a concept of surgical robot shown in Figure 1 with solid lines. The robot does not hold the back end of the surgical instrument but holds it right above of the trocar port so that it has the same operation range of the tip of the instrument as an arm type robot by smaller motion. In Figure 1, the portion of light gray expresses the space occupied with an arm type robot, and the portion of deep gray expresses that of the small surgical robot. The smaller space occupancy of surgical robot is, the larger the surgeon and the assistants can move in the operationg room. We call these small surgical robot which move right above of the trocar port, Active Trocar. The Active Trocar is installed in arbitrarily positions with a passive positioner which has six degree-of-freedom. It enables to use the range of operation of the Active Trocar effectively by making the initial position of it adapted for each operation method. On the basis of the abovementioned concept, we designed a surgical robotic device. The designed robotic device consists of two parts. One is the High Stiffness Multi Degree-of-Freedom Active Forceps that has three degree-of-freedom which determines the posture at the tip of an surgical instrument, and the other is the Active Trocar that has three degree-of-freedom which determines the position of the end-effector in the abdominal cavity. To miniaturize a surgical robot system, we set the specification for the slave robot as follows:

Degree-of-Freedom: A slave robotic device for the endoscopic surgery is required to have at least six degree-of-freedom for dexterity and ease of use for surgeons. We designed the robot with a simple six degree-of-freedom mechanism as shown in Figure 2. One additional degree-of-freedom is for the function of end-effectors on the tip of the surgical instrument(e.g. grasping, scissoring etc.). 


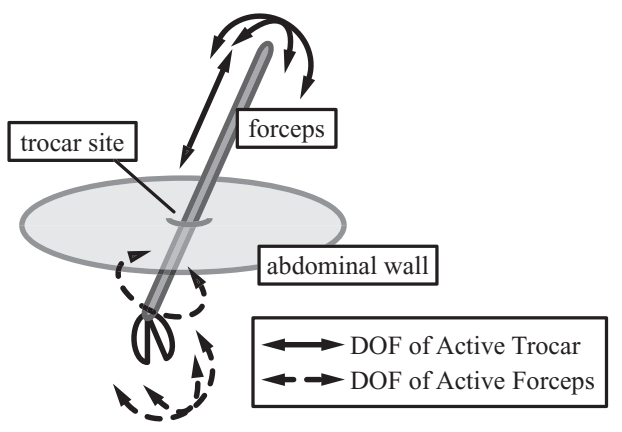

Fig. 2. Degree-of-Freedom of the Surgical Robic System

Field of Activity: The robot shall have the range of operation of 45 [degree] or more from its initial position to all the directions. If the initial position of the robot can be set up arbitrarily, it is considered that it is enough for the space where an endoscopic surgery is performed.

Size of the Robot System: The Active Trocar needs the small amount of motion compared with an arm type robot because of its concepts, and it can be turned into a small robotic system by intensive arrangement of actuators and mechanical composition parts. The size of the Active Trocar must be small enough to be arranged at least 3 bodies on the abdominal wall.

Sterilizability: Surgical instruments should be sterilizable or disposable so that they may not become the infection route. Since the actuator section, including motors, ball screws, and linear guides, is unsterilizable, the contact of the actuator section and surgical instruments which is set on the robot should be avoided. The portion of the robotic system which contact the patient's body is sterilizable, and the whole system is isolated with the patient's body with a drape.

\subsection{Active Trocar}

Figure 3 shows the Active Trocar. The mechanism is based upon the mechanism of the Additional Wrist Mechanism 5. It consists of two actuated rotational joints with DC-servo motors and the Harmonic Drives which has 1/100 reduction ratio and one linear motion mechanism with DC-servo motor, a ball screw and a linear guide to realize three degree-of-freedom of position of the tip of a surgical instrument in the abdominal cabity. Figure 4 shows the mechanism of the Active Trocar. The closed kinematic chain which consists of Link A, Link B, Link E and Link F in Figure 4 restricts the sergical instrument on the Active Trocar to rotate around a certain point shown as point $\mathrm{P}$ in Figure 4 The kinematic chain is actuated with the rotational joint 2 , and it has the flexibility of two degreeof-freedom centering on the point $\mathrm{P}$ by having a rotation mechanism expressed rotational joint 1 in Figure 4. Arranging the Active Trocar so that the point to be the trocar port, it does not damage the abdominal wall with excessive forces. 


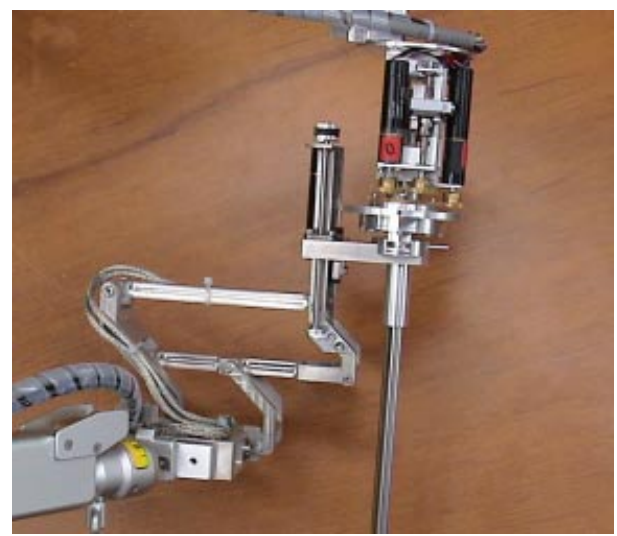

Fig. 3. The Active Trocar with Closed Kinematic Chain

In addition to these rotation mechanisms, three degree-of-freedom of position at the tip of the surgical instrument is decided according to the linear motion mechanism by which the insertion length of the instrument to the abdominal cabity is decided.

The size of designed Active Trocar is miniaturized with the concept of intensive arrangement of the actuators and mechanical parts, and the weight of the robotic device is also light and is approximately $630[\mathrm{~g}]$. Figure 5 shows the size of Active Trocar.

\subsection{Multi-degree-of-freedom Active Forceps}

Most of the typical active forceps adopt wire driven mechanisms. Wire drive active forceps realize a compact and lightweight mechanism, while the elasticity of the wire causes low stiffness of the end-effector, the stretching and friction of the wire cause the low reliability of the system. Therefore we adopt link driven mechanisms, which realizes high stiffness, reliability and responsiveness. Figure 6 shows the mechanism of the active forceps shown as one degree-of-freedom of bending model. There are two links, one is fixed and the other slides along the axis of forceps. The model can be extended to the two degree-of-freedom of bending in the three dimensional space. We adopt it to the mechanism of the active forceps. In addition to these two degree-of-freedom, the active forceps has one degree-of-freedom of rotation, so that it determines the posture at the tip of an surgical instrument. As is mentioned in the section 2.1, the active forceps which contacts the patient's body should be sterilizable. Therefore the forceps section is designed detachable from the actuator section, and the actuator section should be covered with a clean drape.

Figure 7 shows the forceps section and the slide block on the tip and the bottom of the Active Forceps. It consists of three blocks, each of them is the devided part of a cylinder. The cut of each block has hooks so that they do not come apart, we call this mechanism "Jigsaw Block Slide". To decrease the 


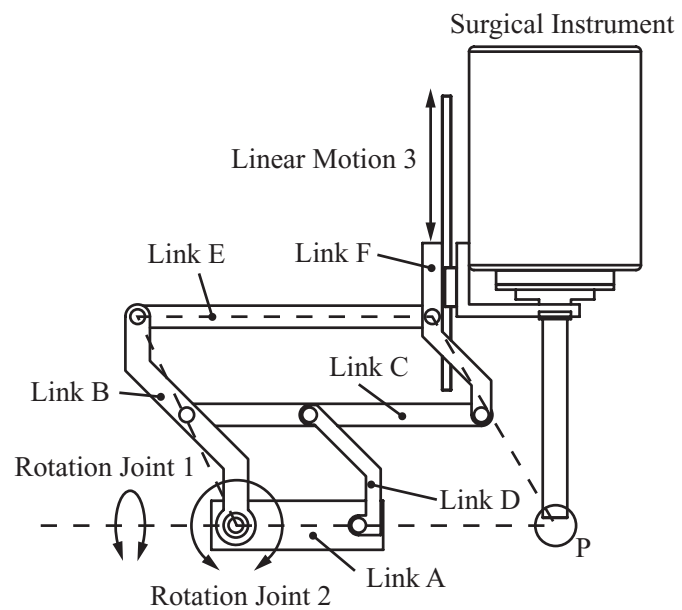

Fig. 4. Mechanism of Active Trocar with Closed Kinematic Chain

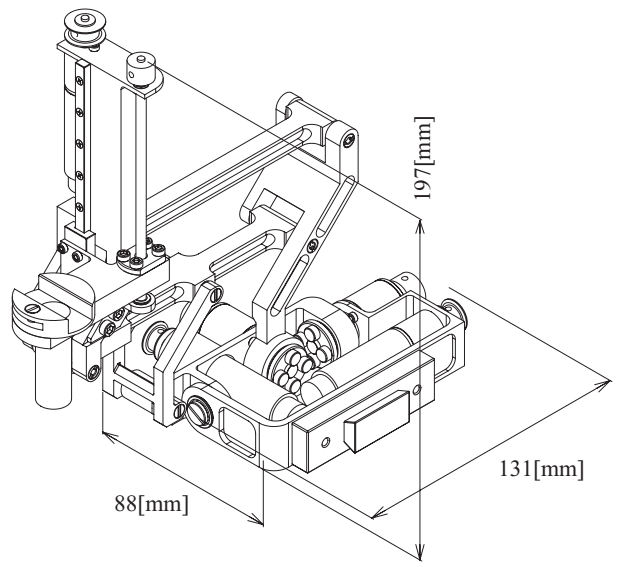

Fig. 5. Size of Active Trocar with Closed Kinematic Chain

friction between each block, Jigsaw Block Slide is made of Silicolloy ${ }^{\mathrm{TM}}$ (Japan Silicolloy Industry Co., Ltd.) which is a kind of stainless steel includes as much as $4 \%$ silicon and has small friction for sliding. The diameter of forceps part is currently $10[\mathrm{~mm}]$ which is the standard size of endoscopes. The links are driven by linear motion mechanisms consist of 2.5[W] DC-servo motors, ball screws and linear guides. The length of the active forceps is currently $300[\mathrm{~mm}]$.

Although the power of actuators was efficiently transmitted with the Jigsaw Block Slide, the stiffness and strength of the mechanism were not satisfactory. The diameter of the endeffector which is shown in Figure 7 is $0.2[\mathrm{~mm}]$ although the diameter of the forceps is $10[\mathrm{~mm}]$. By modifying the structure of the platform and reducing the number of mechanical elements, we designed another active forceps as shown on the left in Figure 8 compared with the present one on the 

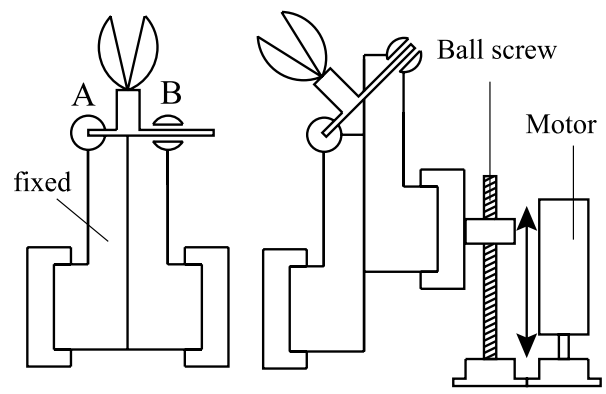

Fig. 6. The Mechanism of Active Forceps Illustrated in One Degree-of-Freedom

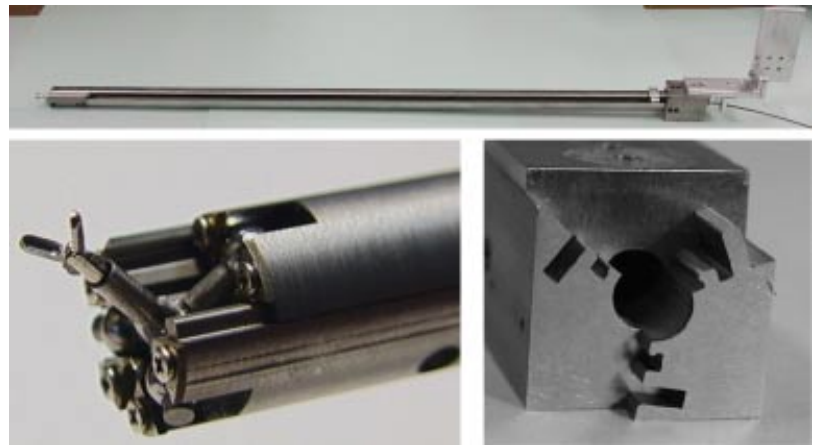

Fig. 7. The whole forceps part (above), The Tip (left) and the Bottom (right) Blocks of the Active Forceps with Biopsy Forceps

right. It uses Jigsaw Block Slide too, while its endeffctor is larger and has higher rigidity. The tip mechanism is changed so that the end plate might be larger. We call the mechanism the Triped Platform. Figure 9 shows the outline of it. The volume of the drive part of the second prototype is $60 \%$ of that of the first prototype.

\section{Experiments of Master-Slave Control}

We set up a master-slave control surgical robotic system for endoscopic surgery. It consists of a master device and a slave robot. We adopt PHANToM DESKTOP $^{\text {тм }}$ (SensAble Technologies Inc.) as the master device which has six axes of measurable joints and three axes of haptic force feedback. The haptic sensation could be used to feedback the force interaction at the end-effector of the slace robot, or to establish haptic communication between the surgeon and the surgical navigation system. However, the current system has no force sensing device. The slave robot consists of the Active Trocar and the Multi Degree-ofFreedom Actuive Forceps, so that it has six degree-of-freedom at the endeffector. We evaluated the operativity of the system by in vivo experiments as shown in 

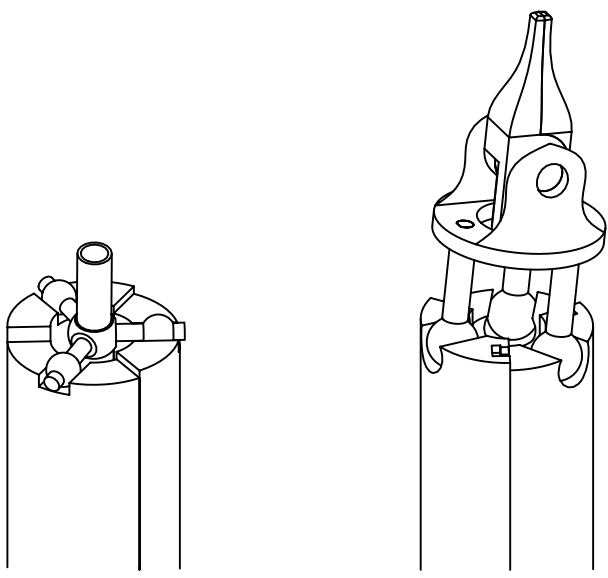

Fig. 8. The Active Forceps with Triped Platform
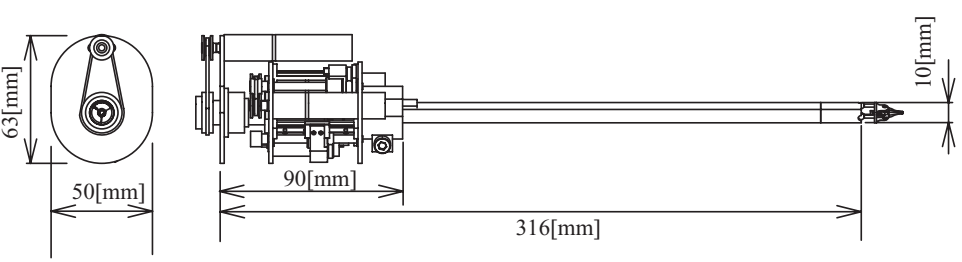

Fig. 9. Outline of the Second Prototype

Figure 10. The result of the experiment is shown in Figure 11. Three upper graphs express the response to the reference data from master device in each axes, and the lower graphs express the error. Although some big errors are seen in each axes, these are because the speed of the linear motion mechanism on the Active Trocar, and it is solvable by changing the pitch of the ball screw.

\section{Conclusions}

The result of this paper is summarized in the following points:

1. We introduced the concept of the Active Trocar that realizes miniaturization of the surgical robotic device.

2. We developed the surgical robot system including Active Trocar and the Multi Degree-of-Freedom Active Forceps.

Acknowledgements: This work was supported through "Development of Surgical Robotic System" (PI: Prof. Ichiro SAKUMA) under the Research for the Future Program, the Japan Society for the Promotion of Science, and through "Improving Manipulatability by Enhansment of Harmonic Scalpel for Laparoscopic Surgery" (PI: Prof. Minoru HASHIMOTO) under the grant-in-aid for scientific research (B). 


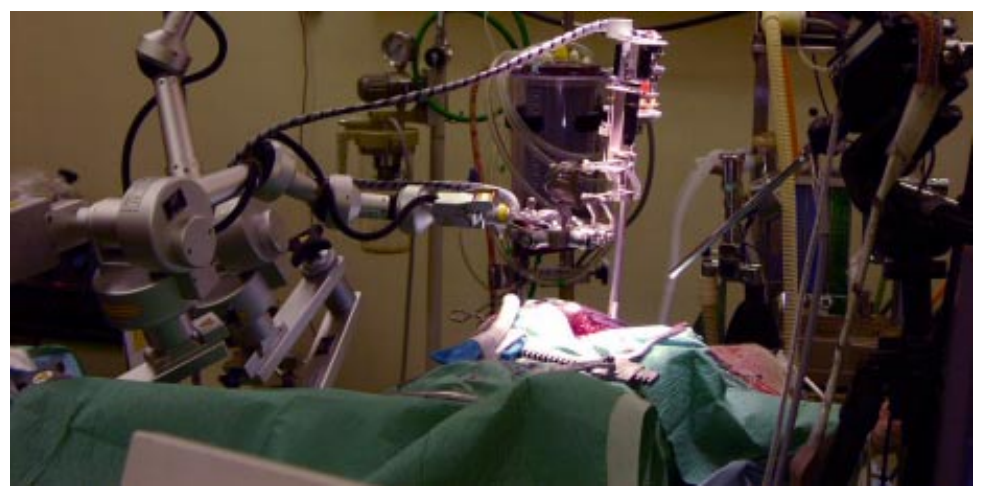

Fig. 10. In vivo Experiment on the Pig
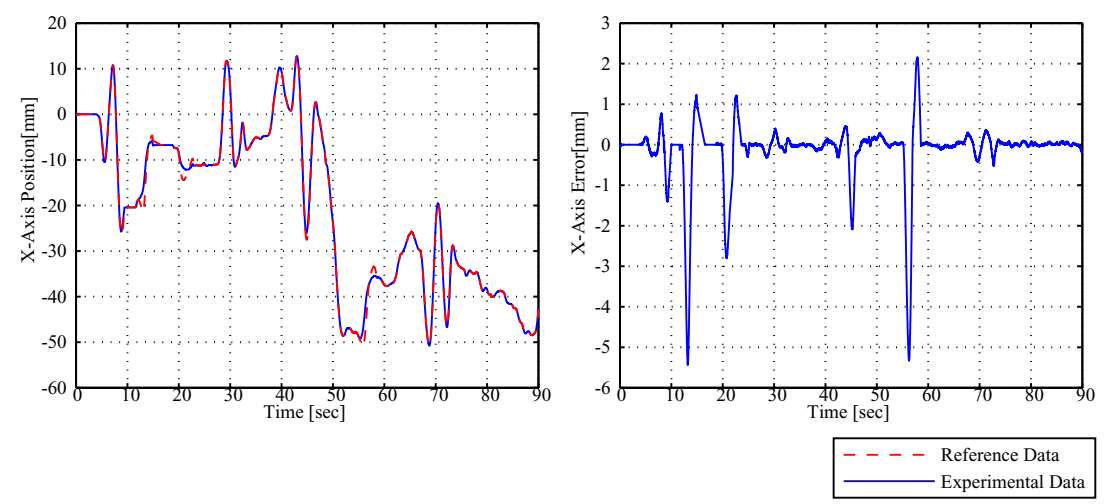

Fig. 11. The Response of the Slave Robot in the in vivo Experiment

\section{References}

1. Guthart G.S. and salisbury J.K. "The Intuitive ${ }^{\mathrm{TM}}$ Telsurgery System: Overview and application". Proc. of the 2000 IEEE International Conference on Robotics 8 Automation, Vol. 1, PP. 618-621, 2000.

2. Reichenspurner H. Damiano R.J., Mack M., Boehm D.H., and Detter C. Gulbins H. "Use of the voice-controlled and computer assisted surgical system ZEUS for endoscopic coronary artery bypass grafting". J. Thorac Cardiovasc Surg., Vol. 118, No. 1, 1999.

3. Nakamura Y., Kishi K. and Kawakami H. "Heartbeat synchronization for robotic cardiac surgery". Proc. of the 2001 IEEE International Conference on Robotics 85 Automation, pp. 2014-2019, 2001.

4. Mitsuhiro H. and Nakamura Y. "Heartbeat syncronization for robotic cardiac surgery". Proc. of the 2001 IEEE International Conference on Robotics 83 Automation, pp. 1543-1548, 2001.

5. Hanafusa H. and nakamura Y. "Autonomous Trajectory Control of Robot Manipulators". Robotics Research: The First International Symposium, pp. 863-882, 1984. 